RENDICONTI DEL CIRCOLO MATEMATICO DI PALERMO

Serie II, Tomo LV (2006), pp. 223-240

\title{
ON A STOCHASTIC DISEASE MODEL WITH VACCINATION
}

ELISABETTA TORNATORE - STEFANIA MARIA BUCCELLATO - PASQUALE VETRO

In memory of Benedetto Pettineo

\begin{abstract}
We propose a stochastic disease model where vaccination is included and such that the immunity isn't permanent. The existence, uniqueness and positivity of the solution and the stability of disease free equilibrium is studied. The numerical simulation is done.
\end{abstract}

\section{Introduction.}

In this paper we consider the stochastic differential system

(1)

$$
\left\{\begin{aligned}
d S(t)= & (\mu-\beta S(t) I(t)-(\mu+\phi) S(t) \\
& +\theta V(t)+\lambda I(t)) d t-\sigma S(t) I(t) d W(t) \\
d I(t)= & (\beta S(t) I(t)+\rho \beta V(t) I(t)-(\lambda+\mu) I(t)) d t+\sigma(S(t) \\
& +\rho V(t)) I(t) d W(t) \\
d V(t)= & (\phi S(t)-\rho \beta V(t) I(t)-(\mu+\theta) V(t)) d t \\
& -\sigma \rho V(t) I(t) d W(t)
\end{aligned}\right.
$$

as a stochastically perturbed system of the ordinary deterministic system

$$
\left\{\begin{array}{l}
S^{\prime}(t)=\mu-\beta S(t) I(t)-(\mu+\phi) S(t)+\theta V(t)+\lambda I(t) \\
I^{\prime}(t)=\beta S(t) I(t)+\rho \beta V(t) I(t)-(\lambda+\mu) I(t) \\
V^{\prime}(t)=\phi S(t)-\rho \beta V(t) I(t)-(\mu+\theta) V(t)
\end{array}\right.
$$

that analyzes a disease spread into a population when individuals are vaccinated.

Precisely the population can be in one of three states: susceptible, infective and vaccinated, denoted by $S, I$ and $V$, respectively. In the model we suppose that the average number of contacts per infective per unit time is a constant $\beta$, that in the unit time a fraction $\phi$ of the susceptible class is vaccinated and 
that the vaccination may reduce but not completely eliminate susceptibility to infection. For this reason in the infection rate of vaccinated members is included a factor $\rho, 0 \leq \rho \leq 1$, with $\rho=0$ meaning the vaccine is perfectly effective and $\rho=1$ meaning the vaccine has no effect. We suppose also that the vaccination loses effect at a proportional rate $\theta$, that the immunity isn't permanent and that a fraction $\lambda$ of infective goes back in the susceptible class so the constant $\lambda$ represents the recovery rate of infected people. We assume that the birth occurs in the system with the same rate constant $\mu$ of death. Consequently the total population is constant and the variables are normalized to $N=S+I+V=1$, that is $S(t)+I(t)+V(t)=1$ for all $t \geq 0$. Of course $\mu, \lambda, \phi, \theta, \beta \in \mathbb{R}_{+}$(see [2], [3], [11]).

Recently there has been interest in the analysis of disease models where a vaccination is introduced. Attention has been given to vaccination and treatment policies both in term of different vaccine classes (all/nothing, leaky) and associated costs ([7-9]). Since the disease can go to an endemic level, control strategies to vaccination are usually implemented to eradicate the disease or at least to lower its prevalence to reasonable level. In modelings such strategies the main problem is that the vaccination is not completely efficient. Vaccines may have low efficacy and be leaky (i.e., after a certain time vaccinated individual may have only partial protection from infection). Data support the fact that a vaccine usually wanes, thus providing only temporary protection. Moreover under certain parameter condition the model (2) exhibits a backward bifurcation leading to the existence of multiple endemic equilibria: a stable disease free equilibrium, an unstable endemic equilibrium and a larger stable endemic equilibrium. This result is important in designing vaccination strategies and in this case the success of the strategy depends on the number of individuals that are initially infected.

This paper is organized as follows. In Section 1 we prove the existence, uniqueness and positivity of the solution of the stochastic system and determine a sufficient condition to eradicate the disease. In Section 2 using Lyapunov functions we prove the stability of disease free equilibrium. Finally in Section 3 we use numerical simulation to compare the deterministic and stochastic behavior of the systems (1) and (2).

\section{Formulation of a stochastic SIS model with vaccination and tempo- rary immunity.}

In this section we prove the existence, uniqueness and positivity of the solution. Throughout this paper, we let $W(t)$ denote a one-dimensional Brow- 
nian motion defined on a stochastic basis $\left(\Omega, \mathcal{F},\left(\mathcal{F}_{t}\right)_{t \geq 0}, \mathbf{P}\right)$ and we let $\sigma$ be a positive constant. If $A$ is a vector or a matrix, its transpose is denoted by $A^{T}$. If $A$ is a matrix, its trace norm is denoted by $|A|=\sqrt{\operatorname{trace}\left(A^{T} A\right)}$. We also introduce the notation

$$
\mathbb{R}_{+}^{3}=\left\{x \in \mathbb{R}^{3}: x_{i}>0 \text { for all } i=1,2,3\right\} .
$$

We consider the system (1) with the following initial condition

$$
(S(0), I(0), V(0)) \in \mathbb{R}_{+}^{3}, \quad S(0)+I(0)+V(0)=1 .
$$

It is observed that the total population is constant $S(t)+I(t)+V(t)=1$ for all $t \geq 0$ almost surely (briefly a.s.), in fact summing the equations of the system (1) we obtain

$$
d(S+I+V)=0 .
$$

Thus, if $S(s)>0, I(s)>0, V(s)>0$ for all $0 \leq s \leq t$ a.s., then

$$
0<S(s)<1, \quad 0<I(s)<1, \quad 0<V(s)<1
$$

for all $0 \leq s \leq t$ a.s.

Since the coefficients of the system (1) are locally Lipschitz the following result of local existence of solutions holds.

THEOREM 2.1. (Theorem $1.1 \mathrm{pg} 98$ of [4]). For any given initial condition $(S(0), I(0), V(0)) \in \mathbb{R}_{+}^{3}$ there exists $\tau>0$ and a unique solution $(S(t), I(t), V(t))$ of the system (1) on $t \in[0, \tau)$.

We consider an integer $k_{0}>0$ sufficiently large such that $(S(0), I(0)$, $V(0)) \in\left[\frac{1}{k_{0}}, k_{0}\right]^{3}$. For each integer $k>k_{0}$ we define the stopping time

$$
\tau_{k}=\inf \left\{t \in[0, \tau):(S(t), I(t), V(t)) \notin\left[\frac{1}{k}, k\right]^{3}\right\} .
$$

We shall show that the solution of (1) is positive and global using the idea exposed in [12].

THEOREM 2.2. For any given initial condition $(S(0), I(0), V(0)) \in \mathbb{R}_{+}^{3}$ there exists a unique solution $(S(t), I(t), V(t))$ of the system (1) on $t \geq 0$ and the solution will remain in $\mathbb{R}_{+}^{3}$ with probability 1 , namely $(S(t), I(t), V(t)) \in$ $\mathbb{R}_{+}^{3}$ for all $t \geq 0$ almost surely.

For the proof of the theorem we need the following lemma. 
LEMMA 2.1. Let $(S(t), I(t), V(t))$ be the solution of the system (1), with initial condition (3); then

$$
E\left(\log ^{2} S\left(\tau_{k} \wedge t\right)+\log ^{2} I\left(\tau_{k} \wedge t\right)+\log ^{2} V\left(\tau_{k} \wedge t\right)\right) \leq C(t)
$$

where $C(t)$ is the solution of the Cauchy problem

$$
\left\{\begin{aligned}
y^{\prime}(t)= & \left(2+\rho^{2}\right) \sigma^{2}+2 \sqrt{3}(\beta(1+\rho)+3 \mu+\phi+\theta+\lambda \\
& \left.+\frac{3 \sigma^{2}}{2}\left(1+\rho^{2}\right)\right) \sqrt{y(t)} \\
y(0)= & \log ^{2} S(0)+\log ^{2} I(0)+\log ^{2} V(0) .
\end{aligned}\right.
$$

Proof. Define a $C^{2}$-function, $\Phi: \mathbb{R}_{+}^{3} \rightarrow \mathbb{R}_{+}$by

$$
\Phi(S(t), I(t), V(t))=\log ^{2} S(t)+\log ^{2} I(t)+\log ^{2} V(t) .
$$

The Ito formula shows that

$$
\begin{aligned}
d(\Phi(S(t), I(t), V(t)))=2\left[\left(\frac{\mu}{S}-\beta I-(\mu+\phi)+\theta \frac{V}{S}+\lambda \frac{I}{S}-\frac{\sigma^{2}}{2} I^{2}\right) \log S\right. \\
+\left(\beta S+\beta \rho V-(\lambda+\mu)-\frac{\sigma^{2}}{2}(S+\rho V)^{2}\right) \log I
\end{aligned}
$$

We observe that $(S(t), I(t), V(t)) \in\left[\frac{1}{k}, k\right]^{3}$ for every $t \leq \tau_{k}$, in virtue of stopping time defined in (5). Using (4) we have a.s.

$$
0<S(t)<1, \quad 0<I(t)<1, \quad 0<V(t)<1 .
$$

We set

$$
Y(t)=E\left(\log ^{2} S\left(t \wedge \tau_{k}\right)+\log ^{2} I\left(t \wedge \tau_{k}\right)+\log ^{2} V\left(t \wedge \tau_{k}\right)\right) .
$$

Taking into account (9) and (8) we get

$$
\begin{gathered}
Y(t) \leq Y(0)+\left(2+\rho^{2}\right) \sigma^{2} t+2 \sqrt{3}(\beta(1+\rho)+3 \mu+\phi+\theta \\
\left.+\lambda+\frac{3 \sigma^{2}}{2}\left(1+\rho^{2}\right)\right) \int_{0}^{t} \sqrt{Y(s)} d s,
\end{gathered}
$$

from which it follows that

$$
Y(t) \leq C(t) \quad \forall t \geq 0,
$$


where $C(t)$ is the solution of the Cauchy problem

$$
\left\{\begin{array}{c}
y^{\prime}(t)=\left(2+\rho^{2}\right) \sigma^{2}+2 \sqrt{3}(\beta(1+\rho)+3 \mu+\phi+\theta+\lambda \\
\left.+\frac{3 \sigma^{2}}{2}\left(1+\rho^{2}\right)\right) \sqrt{y(t)} \\
y(0)=\log ^{2} S(0)+\log ^{2} I(0)+\log ^{2} V(0)
\end{array}\right.
$$

and the lemma is proved.

Proof of the Theorem 2.2. By Theorem 2.1 there exist $\tau>0$ and the solution $(S(t), I(t), V(t))$ of the system (1) on $t \in[0, \tau)$. To prove that this solution is global, we need to show that $\tau=\infty$ a.s. . Consider the stopping time $\tau_{k}$ defined in (5). Clearly $\left(\tau_{k}\right)$ is an increasing sequence. Set $\tau_{\infty}=\lim _{k \rightarrow \infty} \tau_{k}$, whence $\tau_{\infty}<\tau$, a.s.. If we can show that $\tau_{\infty}=\infty$ a.s. then $\tau=\infty$ a.s. and consequently the solution $(S(t), I(t), V(t)) \in \mathbb{R}_{+}^{3}$ for all $t \geq 0$ a.s.. If this statement is false, then there are two constants $T>0$ and $\varepsilon \in(0,1)$ such that

$$
P\{\omega \in \Omega: \tau \leq T\}>\varepsilon .
$$

Let $k_{0}>0$ be such that $(S(0), I(0), V(0)) \in\left[\frac{1}{k_{0}}, k_{0}\right]^{3}$; consequently there exists an integer $k_{1} \geq k_{0}$ such that

$$
P\left\{\omega \in \Omega: \tau_{k} \leq T\right\} \geq \varepsilon \quad \forall k \geq k_{1} .
$$

Set $\Omega_{k}=\left\{\omega \in \Omega: \tau_{k} \leq T\right\}$ for each $k \geq k_{1}$, we have $P\left(\Omega_{k}\right) \geq \varepsilon$. Note that for every $\omega \in \Omega_{k}$ there is some component of $\left(S\left(\tau_{k}\right), I\left(\tau_{k}\right), V\left(\tau_{k}\right)\right)$ equal to $k$ or $\frac{1}{k}$ and hence by (7)

$$
\Phi\left(S\left(\tau_{k}\right), I\left(\tau_{k}\right), V\left(\tau_{k}\right)\right) \geq \log ^{2} k .
$$

From (6) we deduce that

$$
C(T) \geq E\left(1_{\Omega_{k}} \Phi\left(S\left(\tau_{k}\right), I\left(\tau_{k}\right), V\left(\tau_{k}\right)\right) \geq \varepsilon \log ^{2} k\right.
$$

where $1_{\Omega_{k}}$ is the characteristic function of $\Omega_{k}$. Letting $k \rightarrow \infty$ leads to the contradiction

$$
\infty=C(T)<\infty
$$

so we must have $\tau=\infty$ a.s..

Theorem 2.2 and Lemma 2.1 show that $Q=[0,1] \times[0,1] \times[0,1]$ is the invariant set of the solutions of the system (1). We use this observation to find a sufficient condition for disease eradication. In virtue of Ito formula the second equation of system (1) is written as

$$
\begin{aligned}
d \log I(t)= & \left(\beta(S(t)+\varrho V(t))-(\lambda+\mu)-\frac{\sigma^{2}}{2}(S(t)+\varrho V(t))^{2}\right) d t \\
& +\sigma(S(t)+\varrho V(t)) d W
\end{aligned}
$$


LEMMA 2.2. Let $(S(t), I(t), V(t))$ be the solution of the system (2) with initial condition (3). If

$$
\frac{\beta}{\sigma \sqrt{2(\lambda+\mu)}}<1
$$

holds, then we have

$$
\lim _{t \rightarrow \infty} \log I(t)=-\infty \quad \text { a.s.. }
$$

Proof. We observe that for every $t>0$

(13) $\beta(S(t)+\varrho V(t))-(\lambda+\mu)-\frac{\sigma^{2}}{2}(S(t)+\varrho V(t))^{2} \leq \frac{\beta^{2}}{2 \sigma^{2}}-(\lambda+\mu)$.

From (10) and (12), being $S(t)+\rho V(t)<1 \forall t>0$, we have

$$
d \log I(t) \leq\left(\frac{\beta^{2}}{2 \sigma^{2}}-(\lambda+\mu)\right) d t+\sigma d W .
$$

Using (11) and $\lim _{t \rightarrow+\infty} \frac{W(t)}{t}=0$, we obtain as $t \rightarrow+\infty$

$$
\log I(0)+\left(\frac{\beta^{2}}{2 \sigma^{2}}-(\lambda+\mu)\right) t+\sigma W(t) \rightarrow-\infty \quad \text { a.s.. }
$$

Thus we have

$$
\lim _{t \rightarrow+\infty} \log I(t)=-\infty, \quad \text { a.s.. }
$$

\section{Stability of disease free equilibrium.}

As in the deterministic case, the system (1) has the disease free equilibrium (13) $E_{0}=\left(S_{0}, 0, V_{0}\right)$ where $S_{0}=\frac{\mu+\theta}{\mu+\theta+\phi}, V_{0}=\frac{\phi}{\mu+\theta+\phi}$.

We denote by

$$
R_{0}=\frac{\beta}{\mu+\lambda} \quad \text { and } \quad R_{\phi}=R_{0}\left(S_{0}+\rho V_{0}\right)=R_{0}\left(\frac{\mu+\theta+\rho \phi}{\mu+\theta+\phi}\right)
$$

the basic reproduction number (see [1]) and the basic reproduction number in a population in which a proportion $\phi$ has been vaccinated, respectively.

In this section we prove the stability of the disease free equilibrium using definitions and theorems introduced in [6] ( see also [5]).

We recall definitions of equilibrium states of a stochastic differential equation. Consider the following $n$-dimensional stochastic equations system

$$
d X(t)=f(t, X(t)) d t+g(t, X(t)) d W(t)
$$


where $f(t, x)$ is a function in $\mathbb{R}^{n}$ defined in $\left[t_{0},+\infty\left[\times \mathbb{R}^{n}\right.\right.$, and $g(t, x)$ is a $n \times m$ matrix, $f, g$ are locally Lipschitz functions in $x$ and $W(t)$ is an $m$ dimensional Wiener process.

DEFINITION 3.1. The stochastic process $X(t)=\bar{X}$ is a stationary solution of the stochastic system with initial condition $X\left(t_{0}\right)=\bar{X}$ if

$$
f(t, \bar{X})=0, \quad g(t, \bar{X})=0 .
$$

If $\bar{X}=0$, the stationary solution is called a trivial solution.

DEFINITION 3.2. The trivial solution $X(t)=0$ of system (15) is said to be:

1. stable in probability if $\forall \varepsilon>0$ and $s \geq t_{0}$

$$
\lim _{y \rightarrow 0} \mathbf{P}\left\{\sup _{t \in\left[t_{0},+\infty[\right.}|X(t ; s, y)| \geq \varepsilon\right\}=0,
$$

where $X(t ; s, y)$ denotes the solution of system (15) with initial condition $X(s)=y$;

2. asymptotically stable if it is stable in probability and moreover

$$
\lim _{y \rightarrow 0} \mathbf{P}\left\{\lim _{t \rightarrow+\infty}|X(t ; s, y)|=0\right\}=1, \quad s \geq t_{0},
$$

3. globally asymptotically stable if it is asymptotically stable and moreover

$$
\mathbf{P}\left\{\lim _{t \rightarrow+\infty}|X(t ; s, y)|=0\right\}=1, \quad \forall y \in \mathbb{R}^{n} .
$$

Let us denote by $L$ the differential operator associated to (15), defined for a non-negative function $V(t, x) \in C^{1,2}\left(\mathbb{R} \times \mathbb{R}^{n}\right)$ by

$$
L V=\frac{\partial V}{\partial t}+f^{T} \cdot \frac{\partial V}{\partial x}+\frac{1}{2} \operatorname{Tr}\left[g^{T} \cdot \frac{\partial^{2} V}{\partial x^{2}} \cdot g\right] .
$$

Substituting $u_{1}=S-S_{0}, u_{2}=I, u_{3}=V-V_{0}$ into (1), we obtain the system

$$
\left\{\begin{aligned}
d u_{1}(t)= & \left(-\beta\left(u_{1}(t)+S_{0}\right) u_{2}(t)-(\mu+\phi) u_{1}(t)+\theta u_{3}(t)\right. \\
& \left.+\lambda u_{2}(t)\right) d t-\sigma\left(u_{1}(t)+S_{0}\right) u_{2}(t) d W(t) \\
d u_{2}(t)= & \left(\beta\left(u_{1}(t)+\rho u_{3}(t)+S_{0}+\rho V_{0}\right)-\lambda-\mu\right) u_{2}(t) d t \\
& +\sigma\left(u_{1}(t)+\rho u_{3}(t)+S_{0}+\rho V_{0}\right) u_{2}(t) d W(t) \\
d u_{3}(t)= & \left(-\beta \rho\left(u_{3}(t)+V_{0}\right) u_{2}(t)-(\mu+\theta) u_{3}(t)\right. \\
& \left.+\phi u_{1}(t)\right) d t-\sigma \rho\left(u_{3}(t)+V_{0}\right) u_{2}(t) d W(t) .
\end{aligned}\right.
$$


We consider the linearized system

$$
\left\{\begin{aligned}
d u_{1}(t)=\left(-\beta S_{0} u_{2}(t)-\right. & (\mu+\phi) u_{1}(t)+\theta u_{3}(t) \\
& +\lambda u_{2}(t) d t-\sigma S_{0} u_{2}(t) d W(t) \\
d u_{2}(t)=\left(\beta\left(S_{0}+\rho V_{0}\right)-\right. & \lambda-\mu) u_{2}(t) d t \\
& +\sigma\left(S_{0}+\rho V_{0}\right) u_{2}(t) d W(t) \\
d u_{3}(t)=\left(-\beta \rho V_{0} u_{2}(t)-\right. & (\mu+\theta) u_{3}(t) \\
+ & \left.\phi u_{1}(t)\right) d t-\sigma \rho V_{0} u_{2}(t) d W(t)
\end{aligned}\right.
$$

and we prove the following result:

LEMMA 3.1. Let

$$
T=\frac{1}{2} \sigma^{2} \frac{\left(S_{0}+\rho V_{0}\right)^{2}}{\lambda+\mu}
$$

If

$$
R_{\phi}<\min \left\{1-T, \frac{\mu\left(S_{0}+\rho V_{0}\right)}{\lambda+\mu}\right\}
$$

holds, then the disease free equilibrium of (18) is globally asymptotically stable.

Proof. We denote $u=\left(u_{1}, u_{2}, u_{3}\right)$ and we consider the function

$$
V(u)=u_{1}^{2}+\frac{p}{q} u_{2}^{2}+\frac{\theta}{\phi} u_{3}^{2}
$$

where

$$
\begin{gathered}
p=\left(\beta+\sigma^{2}\right)\left(S_{0}^{2}+\frac{\theta}{\phi} \rho^{2} V_{0}^{2}\right)+2 \frac{\lambda^{2}}{\mu}, \\
q=2 \lambda+2 \mu-2 \beta\left(S_{0}+\rho V_{0}\right)-\sigma^{2}\left(S_{0}+\rho V_{0}\right)^{2} .
\end{gathered}
$$

Under condition (20), $V(u)$ is a Lyapunov function and moreover for all $\left(u_{1}, u_{2}, u_{3}\right) \neq(0,0,0)$, we have

$$
\begin{gathered}
L V=-2(\mu+\phi) u_{1}^{2}+\sigma^{2}\left(S_{0}^{2}+\frac{\theta}{\phi} \rho^{2} V_{0}^{2}-p\right) u_{2}^{2}-2 \frac{\theta}{\phi}(\mu+\theta) u_{3}^{2} \\
+2\left(\lambda-\beta S_{0}\right) u_{1} u_{2}+4 \theta u_{1} u_{3}-2 \frac{\theta}{\phi} \beta \rho V_{0} u_{2} u_{3}
\end{gathered}
$$

where $L$ is the operator defined in (16). Taking into account the definition of $p$ and using the following relations:

$$
2\left(\lambda-\beta S_{0}\right) u_{1} u_{2} \leq(\beta+\mu) u_{1}^{2}+\left(\beta S_{0}^{2}+\frac{\lambda^{2}}{\mu}\right) u_{2}^{2},
$$




$$
\begin{gathered}
4 \theta u_{1} u_{3} \leq 2 \phi u_{1}^{2}+2 \frac{\theta^{2}}{\phi} u_{3}^{2}, \\
-2 \frac{\theta}{\phi} \beta \rho V_{0} u_{2} u_{3} \leq \frac{\theta}{\phi} \beta \rho^{2} V_{0}^{2} u_{2}^{2}+\frac{\theta}{\phi} \beta u_{3}^{2},
\end{gathered}
$$

we have

$$
L V \leq-\left[(\mu-\beta) u_{1}^{2}+\frac{\lambda^{2}}{\mu} u_{2}^{2}+(2 \mu-\beta) u_{3}^{2}\right]
$$

Moreover, taking

$$
\begin{aligned}
& a(|u|)=\min \left\{1, \frac{p}{q}, \frac{\theta}{\phi}\right\}|u|^{2}, \\
& b(|u|)=\max \left\{1, \frac{p}{q}, \frac{\theta}{\phi}\right\}|u|^{2}, \\
& c(|u|)=\min \left\{\frac{\lambda^{2}}{\mu}, \mu-\beta\right\}|u|^{2},
\end{aligned}
$$

the thesis follows by Theorem 5.7 of [5], p. 136.

For the stability of the trivial solution of the non-linear system (1), we have the following result.

THEOREM 3.1. If

$$
R_{\phi}<\min \left\{1-T, \frac{\mu\left(S_{0}+\rho V_{0}\right)}{\lambda+\mu}\right\}
$$

holds, then the disease free equilibrium of (1) is asymptotically stable.

Proof. The quantity

$$
\begin{aligned}
& \sqrt{\beta^{2}\left(S-S_{0}\right)^{2} I^{2}+\beta^{2}\left(\left(S-S_{0}\right)+\rho\left(V-V_{0}\right)\right)^{2} I^{2}+\beta^{2} \rho^{2}\left(V-V_{0}\right)^{2} I^{2}} \\
+ & \sqrt{\sigma^{2}\left(S-S_{0}\right)^{2} I^{2}+\sigma^{2}\left(\left(S-S_{0}\right)+\rho\left(V-V_{0}\right)\right)^{2} I^{2}+\sigma^{2} \rho^{2}\left(V-V_{0}\right)^{2} I^{2}}
\end{aligned}
$$

in $] S_{0}-\varepsilon, S_{0}+\varepsilon[\times]-\varepsilon, \varepsilon[\times] V_{0}-\varepsilon, V_{0}+\varepsilon\left[\right.$ is less than $\gamma\left|\left(S-S_{0}, I, V-V_{0}\right)\right|$ with

$$
\gamma=(\beta+\sigma)(1+\rho) \varepsilon
$$

The thesis follows by Theorem 1.1 of [6], p. 248, and Lemma 3.1 . 


\section{Numerical simulations and concluding remarks.}

In this section we use numerical simulations based on the Euler-Maruyama scheme to analyze the stochastic behaviour of the system (1) and to compare it with the deterministic behaviour of system (2).

Let $f(x)$ be a function in $\mathbb{R}^{n}$ defined in $\mathbb{R}^{n}, g(x)$ be a $n \times m$ matrix and $W(t)$ be an $m$-dimensional Wiener process, we consider an $n$-dimensional system of stochastic equations

$$
d X(t)=f(X(t)) d t+g(X(t)) d W(t)
$$

the Euler-Maruyama scheme applied to this equation, on $t \in\{0, \Delta t, \ldots$, $N \Delta t=T\}$ takes the form

$$
X_{\Delta t}(t+\Delta t)=X_{\Delta t}(t)+f\left(X_{\Delta t}(t)\right) \Delta t+g\left(X_{\Delta t}(t)\right) \Delta W(t)
$$

with initial value $X_{\Delta t}(0)=X_{0}$. The $\Delta W(t)=W(t+\Delta t)-W(t)$ represents $N$ independent draws from an $m$-dimensional Normal distribution whose individual components have mean zero and variance $\Delta t$. To generate it using Matlab we refer to [10].

In [13] it is shown that for any finite time and a sufficiently small time step, this numerical scheme will converge to the true solution of (1) provided that a $C^{2}$ function $V: \mathbb{R}_{+}^{n} \rightarrow R_{+}$exists and satisfies the following conditions:

(i) the set $\mathscr{D}(r)=\left\{x \in \mathbb{R}_{+}^{n}: V(x) \leq r\right\}$ is compact for any $r>0$;

(ii) $L V(x) \leq K(1+V(x))$ with a constant $K>0$;

(iii) there exists a positive constant $K_{1}$ such that for all $x, y \in \mathscr{D}(r)$

$$
\begin{gathered}
|V(x)-V(y)| \leq K_{1}|x-y| \\
\left|V_{x}(x)-V_{x}(y)\right| \leq K_{1}|x-y| ; \\
\left|V_{x x}(x)-V x x(y)\right| \leq K_{1}|x-y| .
\end{gathered}
$$

The function $V(x)$ defined in Lemma 3.1 satisfies each of these conditions and so the Euler-Maruyama scheme may be applied to (1).

Moreover we use parameters appropriate for human disease, e.g. pertussis (see e.g. [3], [11]) taking the time unit as a day. Particulary we assume:

$$
\begin{array}{cc}
\lambda=0.04762 & \text { a three week average disease duration } \\
\mu=3.6530 * 10^{-5} & \text { the average lifetime to be } 75 \text { years } \\
\theta=5.4794 * 10^{-4} & \text { the average waning time of the vaccine } \frac{1}{\theta} \text { to be } 5 \text { years } \\
\beta=0.4 & \text { the average number of adequate contacts per infective per day }
\end{array}
$$

and we describe the behaviour as function of the degree of vaccine efficacy $\rho$, the vaccination rate of susceptibles $\phi$ and the stochastic constant $\sigma$. 
Computer simulation of the deterministic model agree well with the results about the existence and stability of the disease free equilibrium and the endemic equilibria. In fact in absence of the disease there is a unique disease free equilibrium $E_{0}\left(S_{0}, 0, V_{0}\right)=\left(\frac{\mu+\theta}{\mu+\theta+\phi}, 0, \frac{\phi}{\mu+\theta+\phi}\right)$ that is globally asymptotically stable if $R_{0}<1\left(R_{0}\right.$ as (14)). If $R_{0}>1$ the endemic equilibria are the solution of (see for example [2]-[3],[11]):

$$
P(I)=A I^{2}+B I+C
$$

where

$A=\rho \beta, \quad B=-\rho \beta+\rho(\mu+\phi+\lambda)+\mu+\theta, \quad C=\frac{(\phi+\mu+\theta)(\lambda+\mu)}{\beta}\left(1-R_{\phi}\right)$ with $R_{\phi}$ as (14).
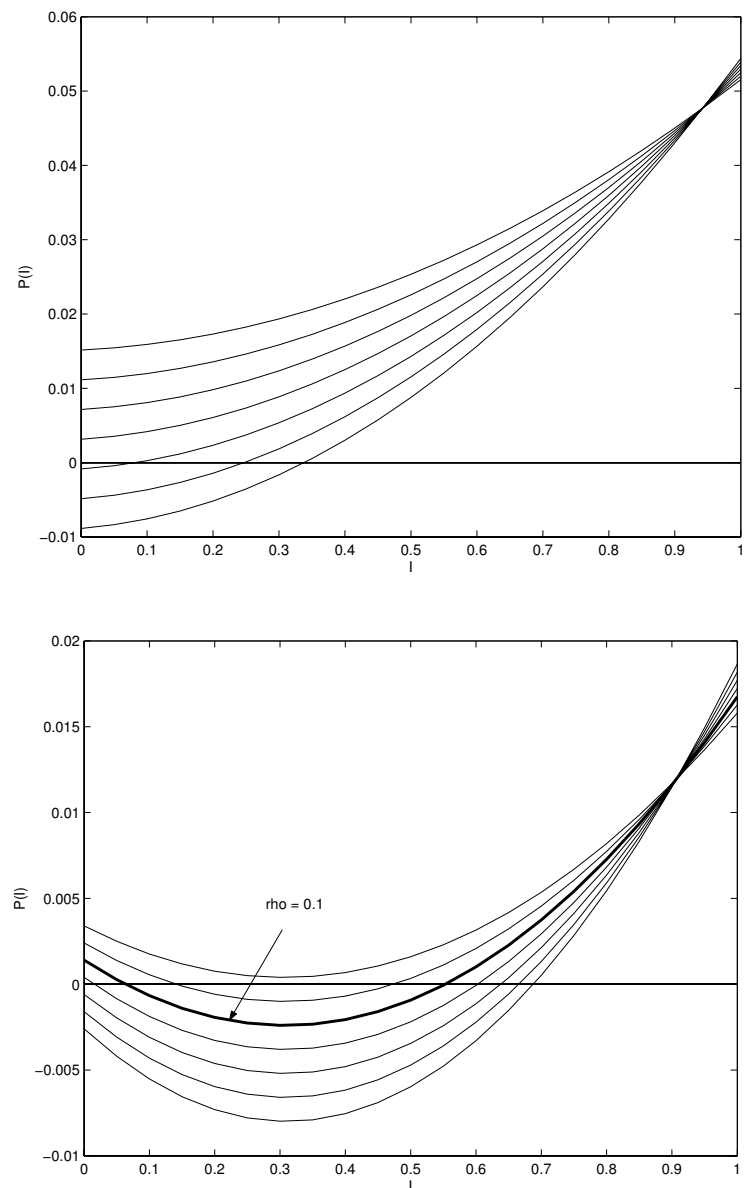

Figure 1 - Roots of $\mathrm{P}(\mathrm{I})$ when $\rho=0.08,0.09, \ldots, 0.14$ and $\phi=0.4, \phi=0.1$ respectively 
Plotting the quadratic $\mathrm{P}(\mathrm{I})$ with increasing values of $\rho$ we visualize as the model can exhibit a backward bifurcation leading to the existence of multiple endemic equilibria and news subthreshold (Figure 1). Particulary for the previous parameter values, if $\rho=0.1$ (90 per cent protection), there is backward bifurcation for a range of $\phi$ values given by $0.0254 \leq \phi \leq 0.1506$ (vaccination of susceptibles on average every 1 to 8 weeks). For example, if $\phi=0.1\left(R_{\phi}=0.8832\right)$ we have the two positive solutions $I_{1}=0.0633$ and $I_{2}=0.5530$ and, then, two endemic equilibria $E_{1}(0.2370,0.0633,0.6997)$ and $E_{2}(0.0810,0.5530,0.3660)$. Thus to describe the behaviour of the deterministic and stochastic systems also near this equilibria, we choose $S(0)=$ $0.0851, I(0)=0.5521, V(0)=0.3628$ as initial data in the simulation. Moreover we use a broken line to represent the deterministic process and a continuous one to represent the stochastic process.

The diagram of bifurcation (Figure 2) shows the roots as function of the parameter $\beta$ and then of $R_{\phi}$. We observe that $R_{c}$ is the value of $R_{\phi}$ at the saddle node bifurcation point where the two values of $I$ coincide with $I=I_{c}=-\frac{B}{2 A}$.

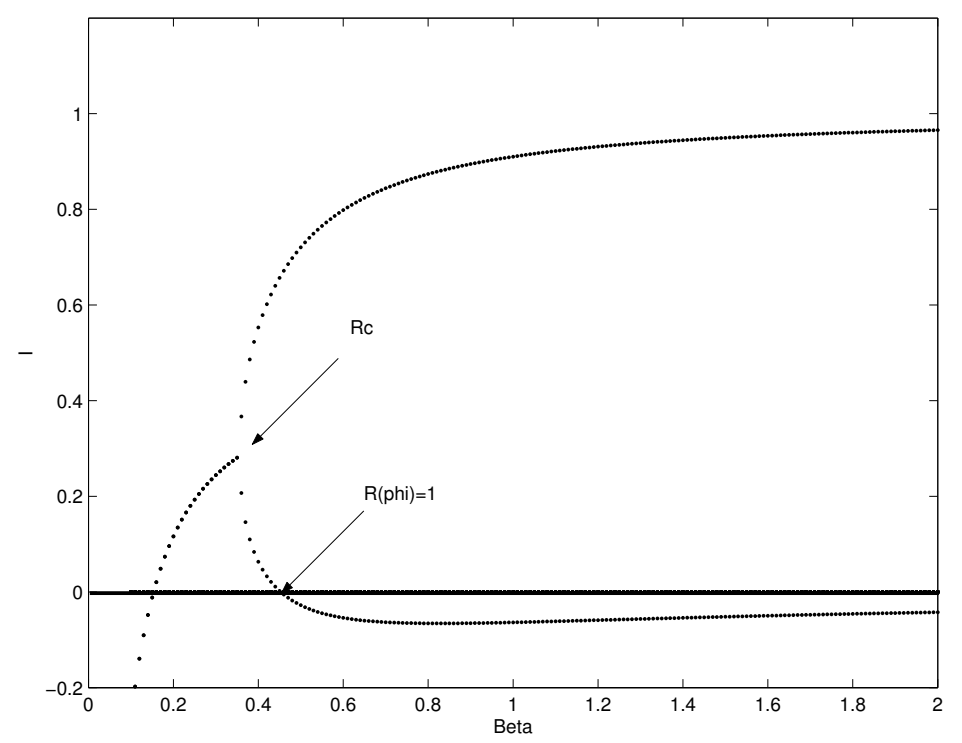

Figure 2 - Roots of $\mathrm{P}(\mathrm{I})$ as function of the parameter $\beta$ and then of $R_{\phi}$ when $\rho=0.1$ and $\phi=0.1$.

About the stability of this equilibria we observe that being the quantity $R_{\phi}$ the number of secondary infectious cases produced by one primary case introduced into a population in which a proportion $\phi$ has been vaccinated, the 
condition $R_{\phi}<1$ is a necessary condition for diseases eradication. However the local stability of $E_{0}$ does not necessarily imply its global stability and this condition is not sufficient.
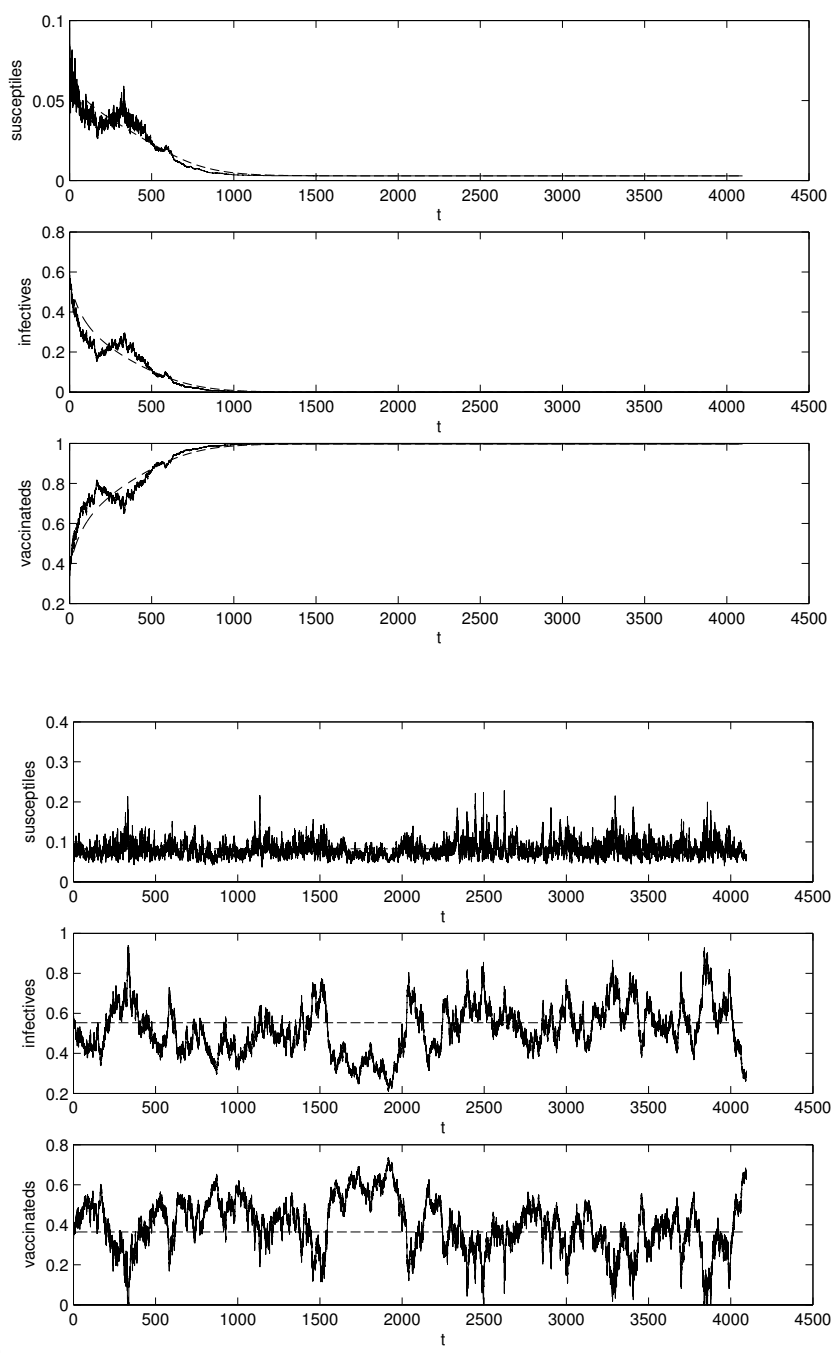

Figure 3 - Number of susceptible, infective and vaccinated population when $\sigma=0.3, \rho=0.1$ and $\phi=0.2000\left(R_{\phi}=0.8614<1-T=0.9901\right), \phi=0.1000\left(R_{\phi}=0.8832<1-T=\right.$ $0.9895)$ respectively.

In fact it is know that for the deterministic system (2) with $V(0) \geq$ $0, I(0)>0$, if $R_{\phi}<R_{c}<1$ then the disease dies out (Figure 3a, Figure 4a and Figure 5a), if $R_{c}<R_{\phi}<1$, then the endemic equilibria $E_{2}$ with larger $I$ is locally asymptotically stable and the endemic equilibria $E_{1}$ with 
smaller $I$ is unstable (Figure 3b, Figure 4b and Figure 5b), if $R_{\phi}>1$, then the unique endemic equilibria $E_{+}$is globally asymptotically stable in $[0,1]^{3} \backslash\{(S, I, V): I=0\}$ (see Theorem 3.1 of [3], p. 487) (Figure 6).
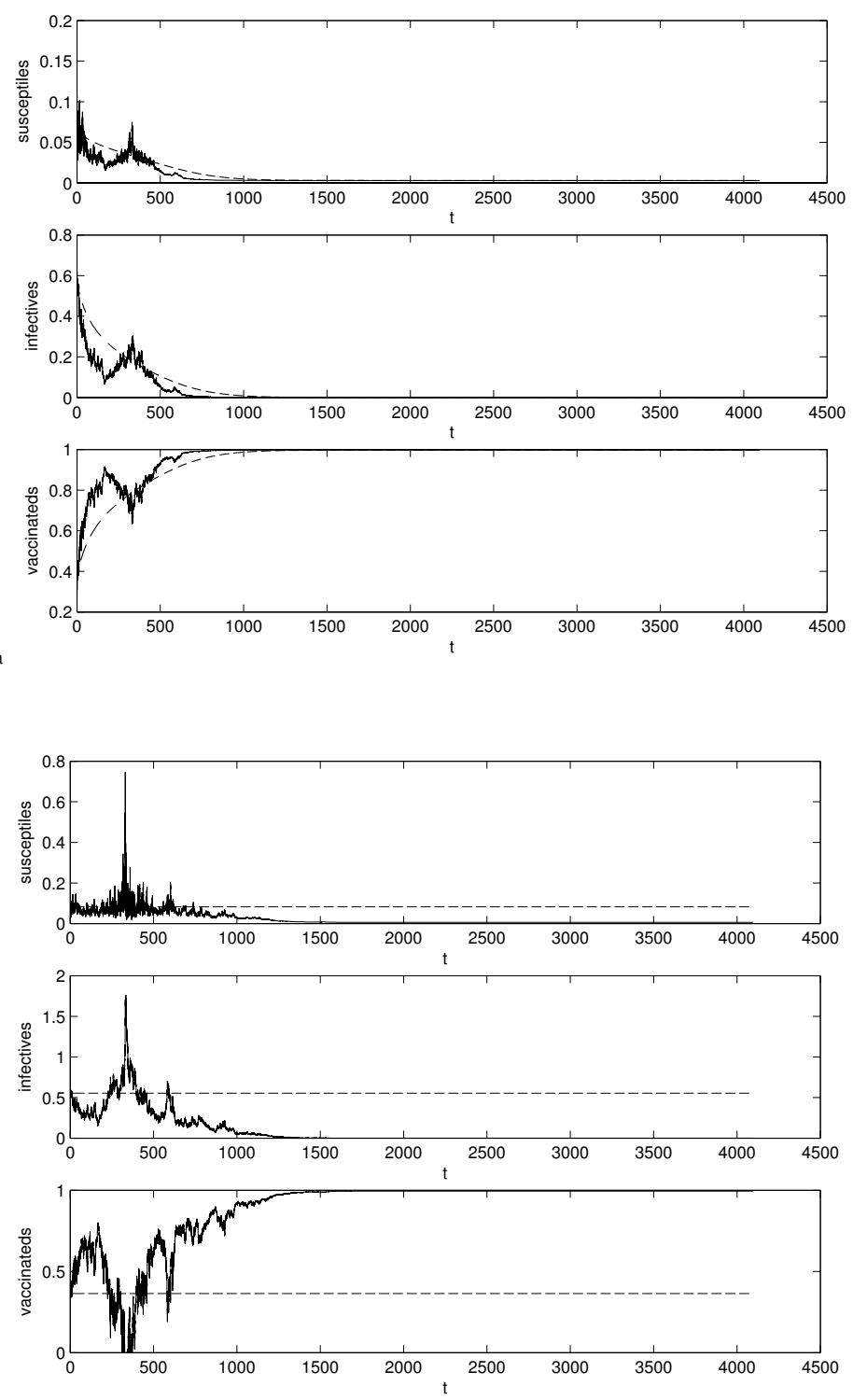

Figure 4 - Number of susceptible, infective and vaccinated population when $\sigma=0.6, \rho=0.1$ and $\phi=0.2000\left(R_{\phi}=0.8614<1-T=0.9582\right), \phi=0.1000\left(R_{\phi}=0.8832<1-T=\right.$ $0.9256)$ respectively. 

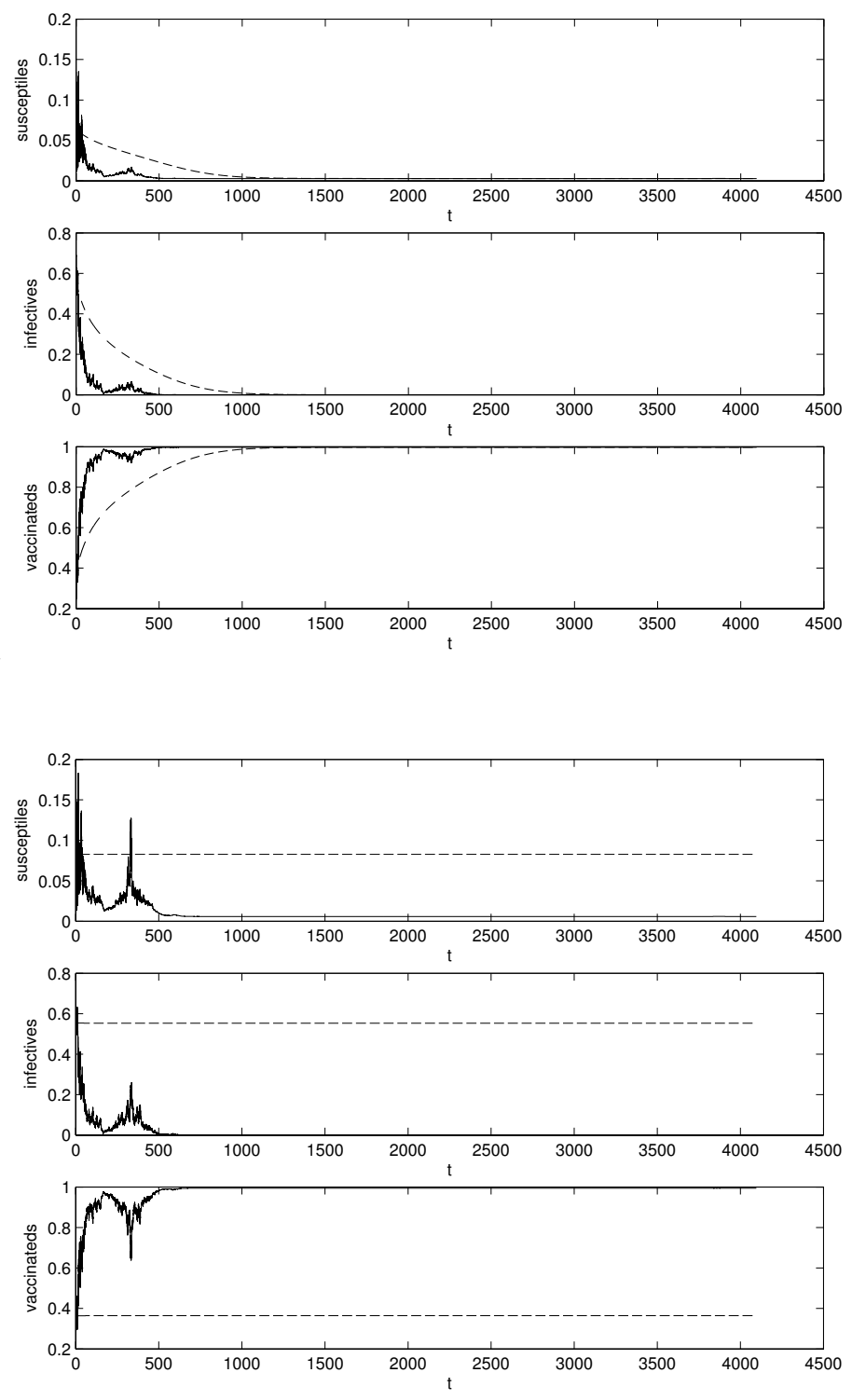

Figure 5 - Number of susceptible, infective and vaccinated population when $\sigma=1.2, \rho=0.1$ and $\phi=0.2000\left(1-T=0.8409<R_{\phi}=0.8614\right), \phi=0.1000\left(1-T=0.8594<R_{\phi}=\right.$ 0.8832 ) respectively.

Numerical simulations of the stochastic model shows that, from the biological point of view, the introduction of a noise in the deterministic model modifies the deterministic threshold $R_{\phi}=1$ of the disease free equilibrium $E_{0}$ giving rise to new threshold $1+T$ ( $T$ as (19)) for $R_{\phi}$. In fact, differently 
that the deterministic model, the disease free equilibrium $E_{0}$ is asymptotically stable for the stochastic model also under the following condition (Figure 6a):

$$
1<R_{\phi}<1+T
$$
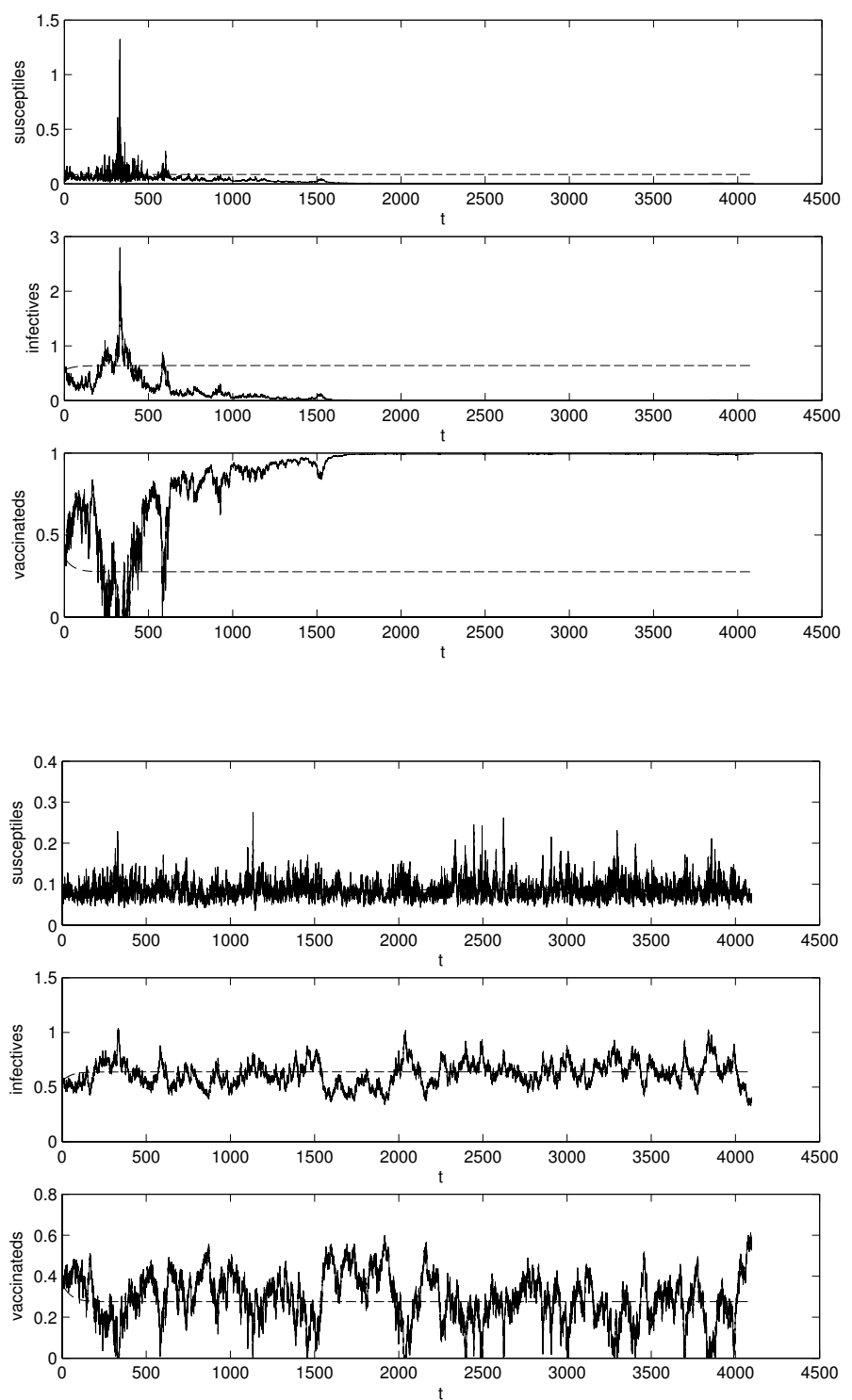

Figure 6 - Number of susceptible, infective and vaccinated population when $\phi=0.1000$, $\rho=0.1200$ and $\sigma=0.8000\left(1<R_{\phi}=1.0501<1+T=1.1051\right), \sigma=0.3000$ $\left(1<1+T=1.0148<R_{\phi}=1.0501\right)$ respectively. 
While if $R_{\phi}>1+T$ then, as the deterministic model, the disease free equilibrium $E_{0}$ is unstable and the solution of system (1) fluctuates around the endemic equilibrium $E_{+}$(Figure 6b).

Finally if $R_{\phi}<1$ the computer simulation shows that the solution of system (1) converges to the disease free equilibrium if there is not a backward bifurcation for the deterministic model (Figure 3a, Figure 4a and Figure 5a) or if there is a backward bifurcation for the deterministic model but $1-T<R_{\phi}<$ 1 (Figure 5b). While, if $R_{\phi}<1-T$ and if there is a backward bifurcation for the deterministic model, we conjecture that there exists a $\sigma_{c}$ value (with the previous parameters value $0.46<\sigma_{c}<0.47$ ) such that, if $\sigma<\sigma_{c}$ the solution of system (1) fluctuates around the biggest endemic equilibrium (Figure $3 \mathrm{~b}$ ) moreover if $\sigma_{c}<\sigma$ the solution of system (1) converges to the disease free equilibrium (Figure $4 b$ ).

\section{REFERENCES}

[1] Anderson R. M., May R. M., Infectious Disease of Humans, Oxford University Press, 1991.

[2] Arino J., McCluskey C. C., Van den Driessche P., Global results for an epidemic model with vaccination that exhibits backward bifurcation, SIAM J. Appl. Math., 64 (2003), 260-276 (electronics).

[3] Arino J., Cooke K. L., Van den Driessche P., J. Velasco Hernándenz, An epidemiology model that includes a leaky vaccine with a general waning function, Discrete Contin. Dyn. Syst., B, 4 (2004), 479-495.

[4] Friedman A., Stochastic differential equations and applications, Academic Press, 1975.

[5] Gard T. C., Introduction to stochastic differential equations. Marcel Dekker, New York, 1988.

[6] Has'minskij R. Z., Stochastic stability of differential equations, Sijthoof \& Noordhoof, Alphen aan den Rijn, The Netherlands, 1980.

[7] Hethcote H. W., The mathematics of infectious disease, SIAM Review, 42 (2000), 599-653.

[8] Hethcote H. W., Qualitative analyses of communicable disease models, Math. Biosci., 28 (1976), 335-356.

[9] Hethcote H. W., Optimal ages of vaccination for measles, Math. Biosci., 89 (1988), 29-52.

[10] Higham D. J., An algorithmic introduction to numerical simulation of stochastic differential equations, SIAM Review, Education Section, 43 (2001), 525-546.

[11] Kribs-Zaleta C. M., Velasco-Hernández J. X., A simple vaccination model with multiple endemic states, Math. Biosci., 164 (2000), 183-201. 
[12] Mao X., Marion G., Renshaw E., Environmental Brownian noise suppresses explosions in population dynamic, Stochastic Process Appl., 97 (2002), 95-110.

[13] Marion G., Mao X., Renshaw E., Convergence of the Euler scheme for a class of stochastic differential equation, Internat. Math. J., 1 (2002), 9-22.

Pervenuto il 4 aprile 2006.

E. Tornatore Dipartimento di Matematica ed Applicazioni, Università di Palermo, Via Archirafi, 34,

90123 Palermo, Italia e-mail: elisa@math.unipa.it

Stefania M. Buccellato Dipartimento di Matematica ed Applicazioni,

Università di Palermo, Via Archirafi, 34,

90123 Palermo, Italia e-mail: bucci@math.unipa.it

P. Vetro

Dipartimento di Matematica ed Applicazioni,

Università di Palermo,

Via Archirafi, 34,

90123 Palermo, Italia e-mail:vetro@math.unipa.it 